\title{
SIMULATION OF MECHANICAL SYSTEM STABILIZATION WITH ACTIVE RESONANCE CONTROLLER
}

\author{
Olena Slavko \\ Kremenchuk Mykhaylo Ostrogradskiy State University, \\ Pervomaiskaya 20, Kremenchuk, Poltava region, Ukraine, 39600, \\ e-mail: slavkolena@gmail.com
}

\begin{abstract}
A simulation model of a mechanical system with a control algorithm based on the active resonance control approach is created. Methodology and virtual laboratory for researches of the simulation models of mechanical systems stabilization based on the active resonance control algorithm are developed. This virtual laboratory allows to research quality and stability of the active resonance algorithm for different types of mechanical systems and external disturbances for various initial conditions and environment parameters. The active resonance control algorithm for the first time is adapted and improved by using of numerical values of velocity and acceleration of a controlled object on each step of a simulation for a system described by a second order differential equation.
\end{abstract}

Keywords: Simulation, control system, active resonance controller, stabilization, identification.

\section{INTRODUCTION}

Automatic controllers are modeled in specialized software like VisSim, SCADA, etc. The main disadvantage of that software is a theoretical disturbances signals forming without a connection with a real control system. On the other hand, a simulation of a mechanical system under an influence of random external disturbances can be executed in such software as Ansys, LS-Dyna and so on. But this software is unable to simulate automatic controllers.

The requirement to join such software abilities appears for modeling of systems which use the active resonance (AR) control approach. The AR control approach has been offered in $[1,2]$. The main idea of this approach is a control signal making based on the equivalent of unknown external disturbances for control system stabilization. The AR method allows to stabilize a control system in case of low a priori information about the control object and external disturbances that is an important practical problem [3].

Abilities of some simulation systems (for example, MATLAB) are well known for temporary information transmission from one system to other by using the OLE- or DDE-technology. But the requirement of the accountability of a simulation real time, model time, its synchronization and unavoidable time delays which are caused by a calculation and data transmission makes an adequate model creation much more complex. One of the active resonance algorithm advantages is its integration with a step-by-step tracking of a system reaction on unknown external disturbances and with a system identification algorithm. This allows to create an adequate controlling signal in a real time.

The purpose of this article is to integrate a controller based on the active resonance control algorithm and a mechanical system simulation with modern CAD-systems, avoiding mentioned disadvantages.

WORKINGMODEL was chosen for this purpose after detailed analysis of CAD-systems abilities and toolboxes. This system has advanced toolboxes for a mechanical systems modeling. Also it has a high integration and an ability of data and models exchange with standard CAD-systems (Cosmos, AutoCad, Kompas and so on). One of the main advantages of WORKINGMODEL is using of the WMB language (Working Model Basic) of the VBA type (Visual Basic Application). This system supports a dynamical controlling of mechanical systems parameters in a real time, ability of a simulation time intervals controlling and temporary calculation during the current time interval and delays controlling in mechanical systems dynamics.

\section{MODEL PARAMETERS}

Consider a simulation model creation of a dynamic system defined by the following 
differential equation:

$$
m \frac{d^{2} x}{d t^{2}}+\lambda \frac{d x}{d t}+k x=f(t)+u(t) \pm m g
$$

where $m-$ controlled object mass, $\lambda-$ environmental resistance coefficient, $k$ - elastic force coefficient, $f(t)$ - external disturbance, $u(t)$ - controlling signal, $x(t)-$ control system reaction (system out-put signal), $g$ - gravity.

This dynamical system is controlled by the active resonance control algorithm. Consider a mechanical system (Fig.1) that is classical for the equation (1).

The controlled object is a sphere that oscillates on a spring. Controlled object properties have been set in appropriate panels before simulation.

Elasticity law $(F=k x)$, elasticity coefficient value $(N / m)$, current and initial spring length $(m)$ at the initial simulation time are set on the spring properties settings panel.

Material, geometrical and mechanical properties, initial positions, initial velocities and accelerations are set on the panel of properties settings for a sphere.

Control signal forming based on the active resonance approach is described in $[1,2]$. Stabilization algorithm of the active resonance approach is the next $[1,2,5]$. During some period of time the controlled process model is made as a controlling signal that is equal to unknown external disturbances. This created equivalent is

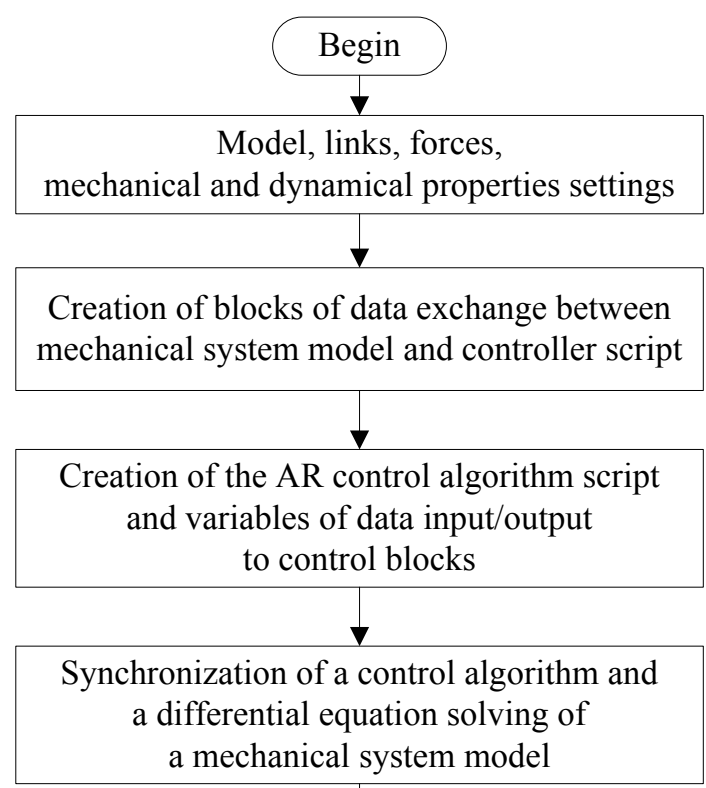

approximated by some analytical expression. Then approximated signal is extrapolated and this extrapolated signal is inverted.

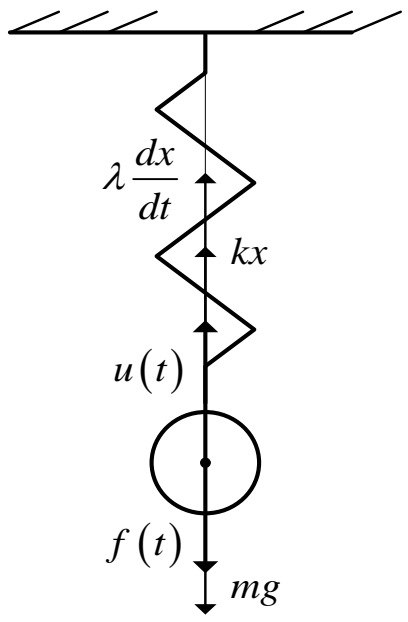

Fig. 1 - Mechanical system scheme

Simulation model creation of a dynamic control system of a mechanical system (1) with the active resonance controller is executed according to the flow chart shown in Fig.2.

Mechanical system simulation model controlling is made according to the general flow chart of a control algorithm (see Fig.3), where $\mathrm{N}$ is a number of steps for an external disturbance equivalent creation according to the active resonance algorithm in this flow chart.

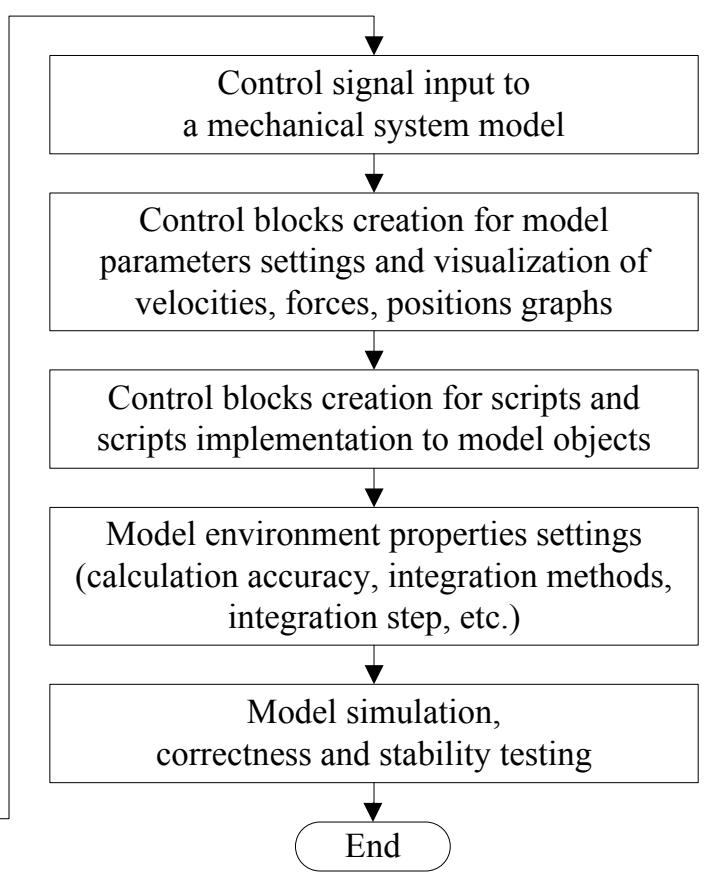

Fig. 2 - Flow chart of the simulation model creation 


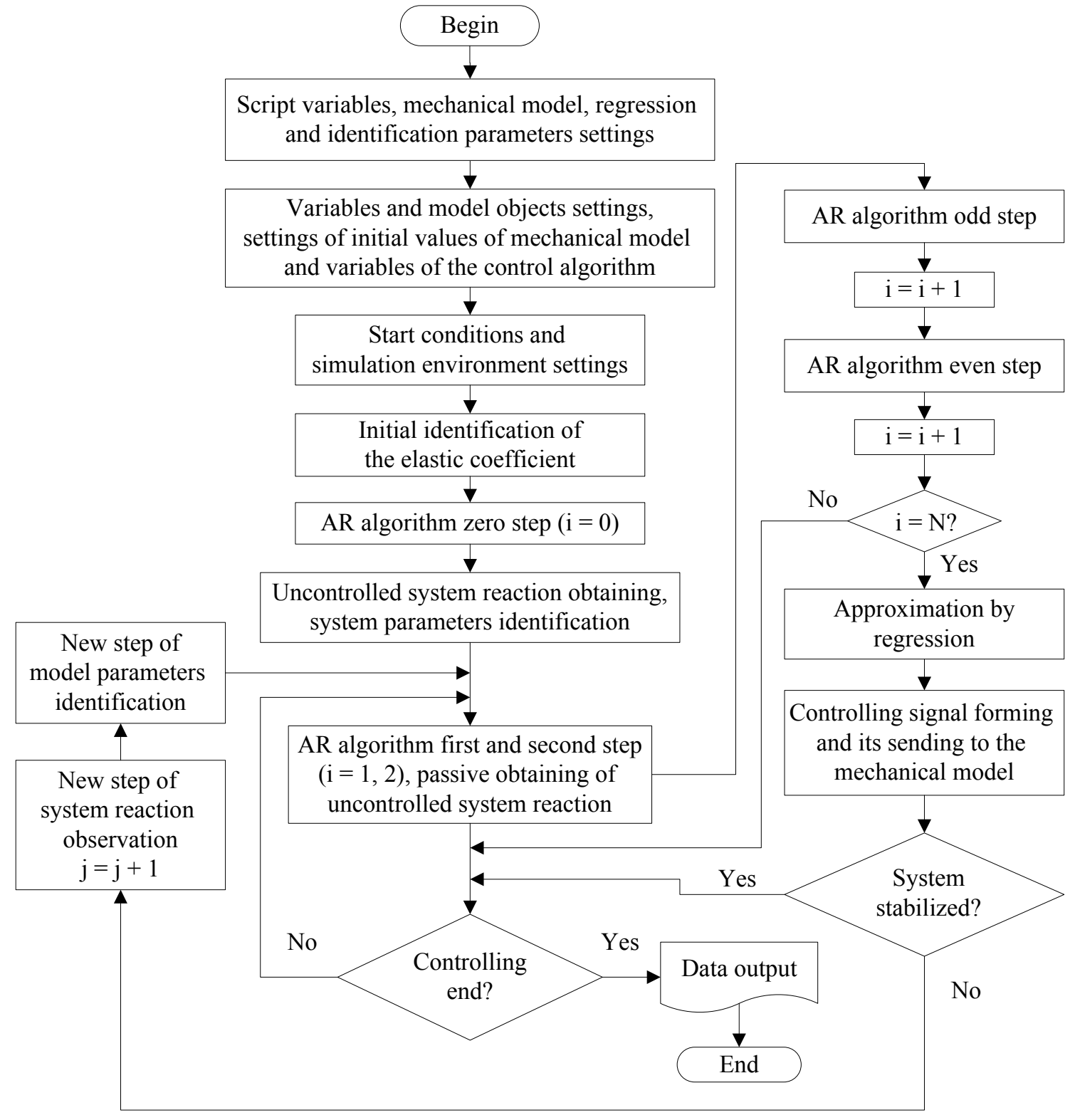

Fig. 3 - General flow chart of the control algorithm

Variables of the environment and the forces functions in a model script have been set after the mechanical system model creation (after visual construction of elements, links, forces, etc.) according to [4]. Parameters of data values exchange from mechanical model to scripts of controller, identification and approximation blocks also have been set.

The example of a virtual laboratory desktop is shown in Fig.4.

Mark "1" shows a fragment of the controller script code in Fig.4. Mark "2" shows a panel for type and direction settings of a gravitational influence on a controlled object. Marks " 3 " and " 4 " are forces properties and connecting parameters panels. Mark "5" is an example of a control signal graph. Marks " 6 " and " 7 " corresponds to the controlled and uncontrolled mechanical system respectively. Mark " 8 " shows a graphs visualization settings panel.

Let us describe labels on the forces properties panel. Label "FRAMES" is time frames, label "DISTe is an external disturbances signal, label "POS" is a current position of a controlled object and label "ARREG" is an active resonance controller signal for this example of a mechanical system.

A synchronization of two models (controllable and uncontrollable) is made for simulation results analysis of a control signal stabilization influence created by the active resonance controller script. So there are two oscillating models on the desktop (marks "6" and "7" in Fig.4). This allows to analyze and compare simulation results. 


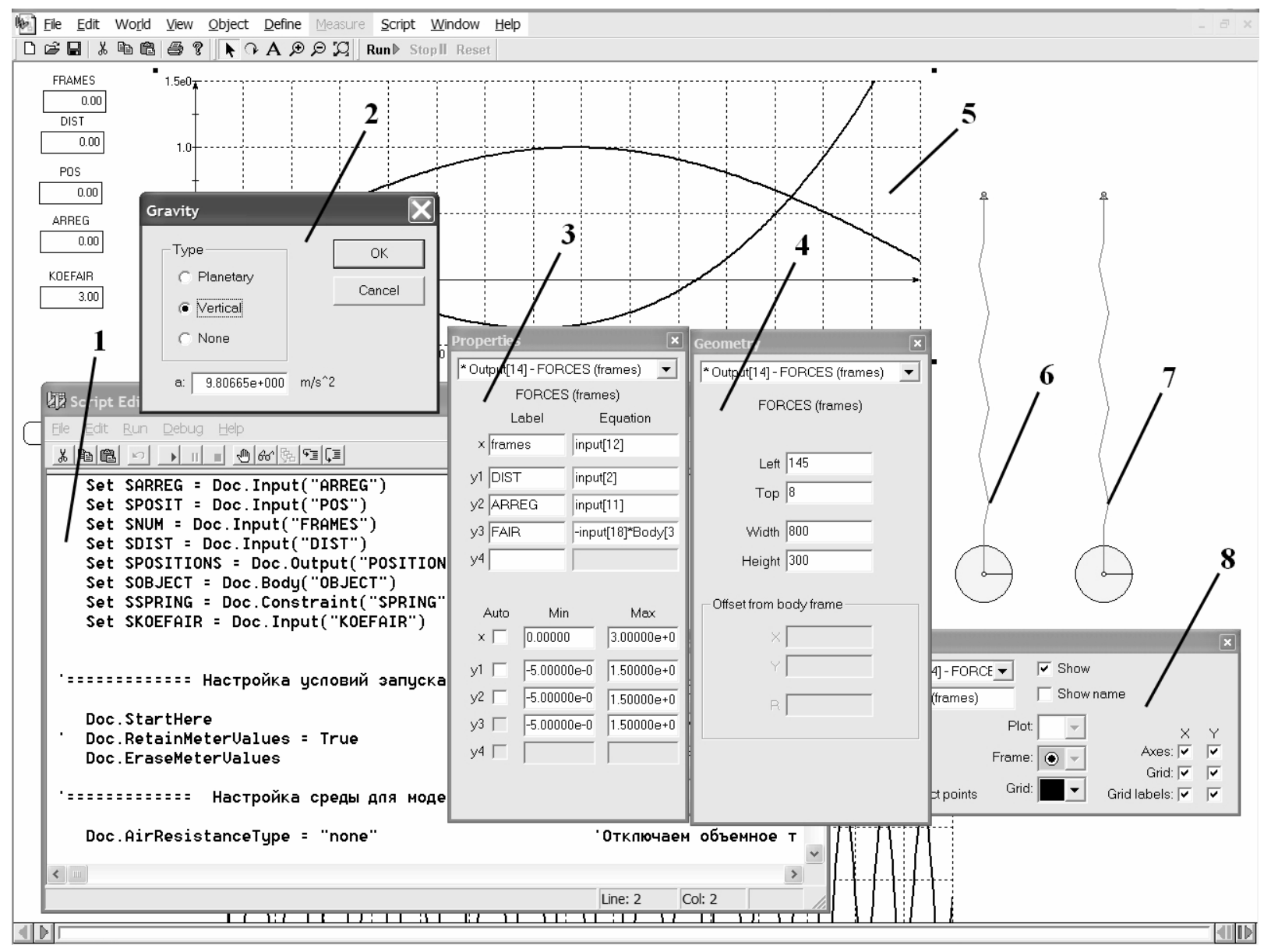

Fig. 4 - Virtual laboratory desktop

\section{SIMULATION RESULTS}

Integration settings (accuracy, methods, etc.) have been done before a mechanical model simulation (see Fig.5).

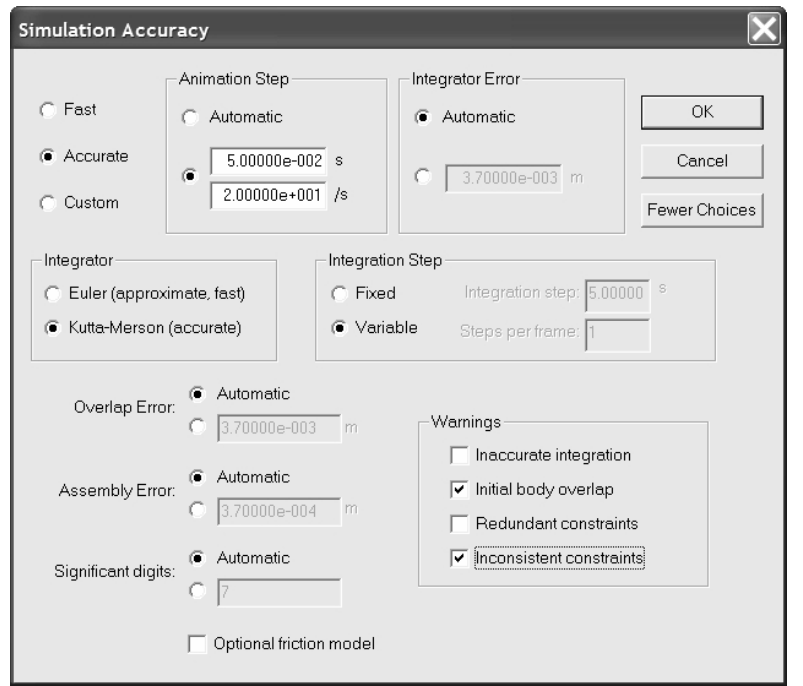

Fig. 5 - Simulation properties settings panel

Kutta-Merson method (which provides accurate integration) or Euler method (which provides approximate but fast integration) with automatic or manual controlling of integration error and with variable or fixed integration step for dynamics system equation integration can be used.

Examples of final graphs of the mechanical system simulation model simulation and investigation are shown in Fig.6-8. The abscissa axis is a time in model frames ( 1 frame $=5 \cdot 10^{-2} \mathrm{~s}$ ) for all graphs. Forces values are shown on the ordinate axis $(N=[\mathrm{m} / \mathrm{s}])$.

The example of a graph of an external disturbance that is unknown for the controlling algorithm and described by a sinusoid in the appropriate script is shown in Fig.6. External disturbances could be described by determinate or random functions of time. Example of the external disturbance script code is shown in Table 1.

Table 1. Disturbance function script code

Function FDist $(i, h)$ As Double
'h - time step, $i-$ time step number
'A - amplitude, $w$-angular frequency
'f - phase shift
FDist $=A^{*} \sin \left(w^{*} h{ }^{*} i+f\right)$
End Function

The control signal is an equivalent of the external disturbance from zero to the 30th frame $(\mathrm{N}=30$ in Fig.3) for this variant of a mechanical system dynamics and the active resonance control algorithm settings. On the next frames (from the 31 frame) the 
control signal is an inversion of the extrapolated signal of the external disturbance equivalence approximated by a third order polynomial regression that is sufficient for an adequate controlling till 180th frame. Thus 30 frames are enough for adequate system stabilization on time period that exceeds an equivalent making time interval in six times. But, as shown in [5], there is an optimal value of time interval of an external disturbance equivalent making. For example, for a harmonic external disturbance this interval should be equal to the $1 / 16$ of a disturbance semiperiod.

Graphs of controlled object velocities and accelerations are shown in Fig.7. These values are computed by a numerical differentiation of a controlled object current position in each frames and used in the active resonance controller script.

Controller script algorithm is based on the active resonance control algorithm $[1,2]$ for a linear object. In this paper modification of the active resonance control algorithm and adaptation to dynamic systems described by a second-order differential equations are made. This improvement was made by a numerical values of a controlled object velocity and acceleration received in a real (simulation) time. So the active resonance control algorithm was implemented for a dynamic system described by the equation (1) for the first time. Simulation data shown in Fig.7 conforms to other graphs and give more accurate representation of a controlled object.

Results of the system simulation with and without a controlling signal are shown in Fig.8. Line 1 is an uncontrolled object oscillation that is caused by a sinusoidal disturbance force in a gravity field at the presence of environment resistance with specified elastic force without taking into account a friction force of the sphere surface. This task is well known and has an exact analytical solution that is equal to the line 1 on the graph in Fig.7.

Line 2 in fig. 8 is a controlled object oscillation in case of the active resonance controller signal influence. Since 30th frame a controlled object position is stabilized and this position is kept within the limits of a permissible error during an adequate extrapolation of the approximated equivalence of an external disturbance signal.

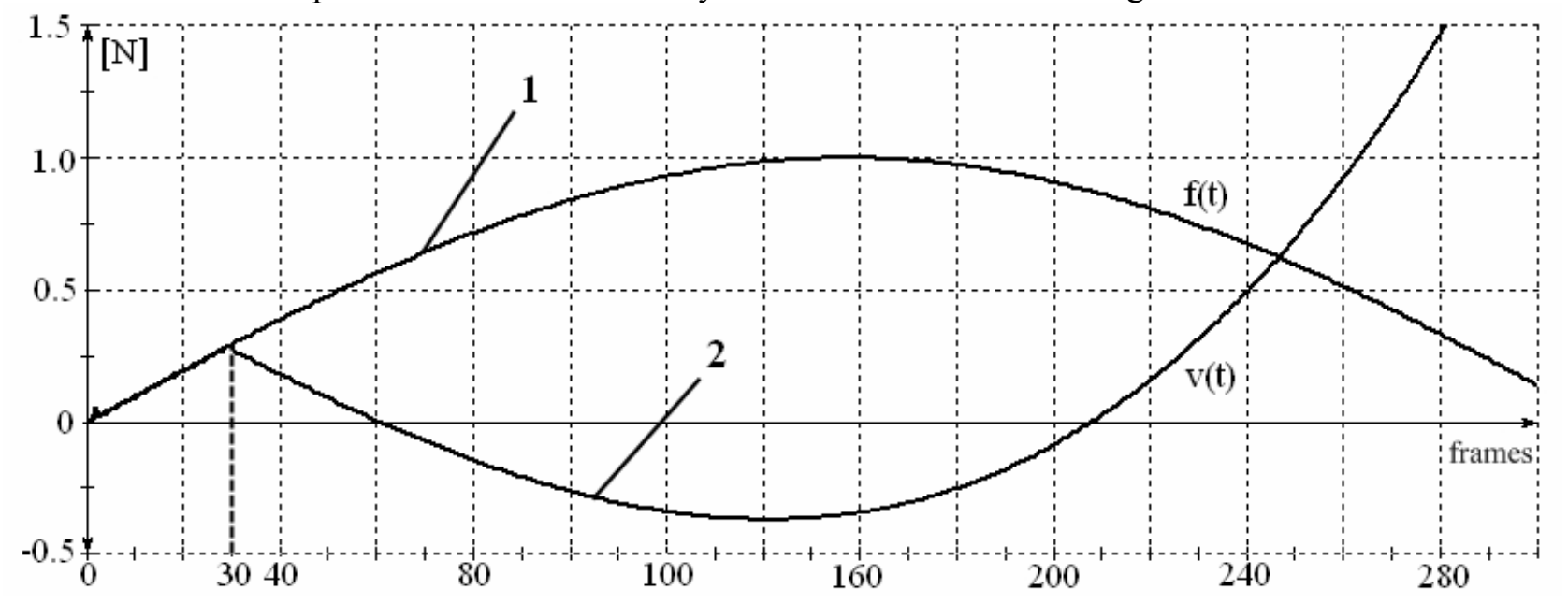

Fig. 6 - Control signal: 1 - external disturbance $f(t), 2$ - control signal $u(t)$

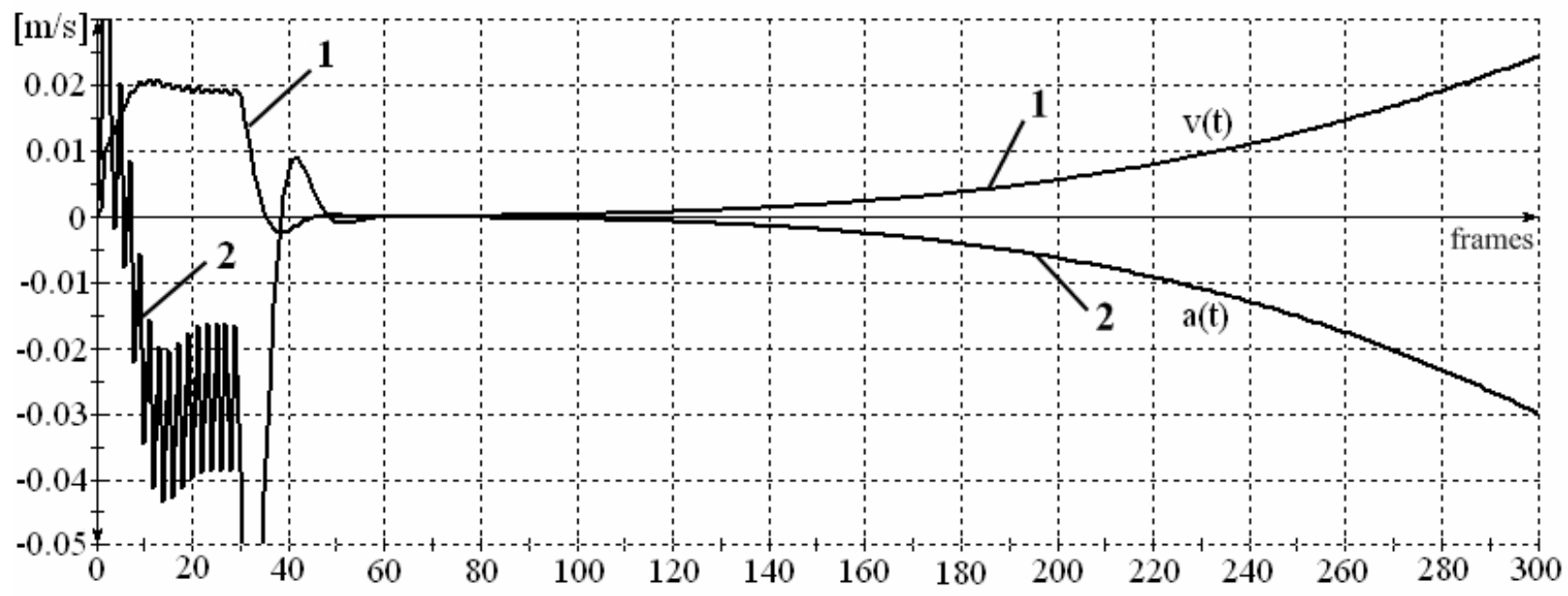

Fig. 7 - Simulation results: 1 - control object velocity v(t), 2 - control object acceleration a(t) 


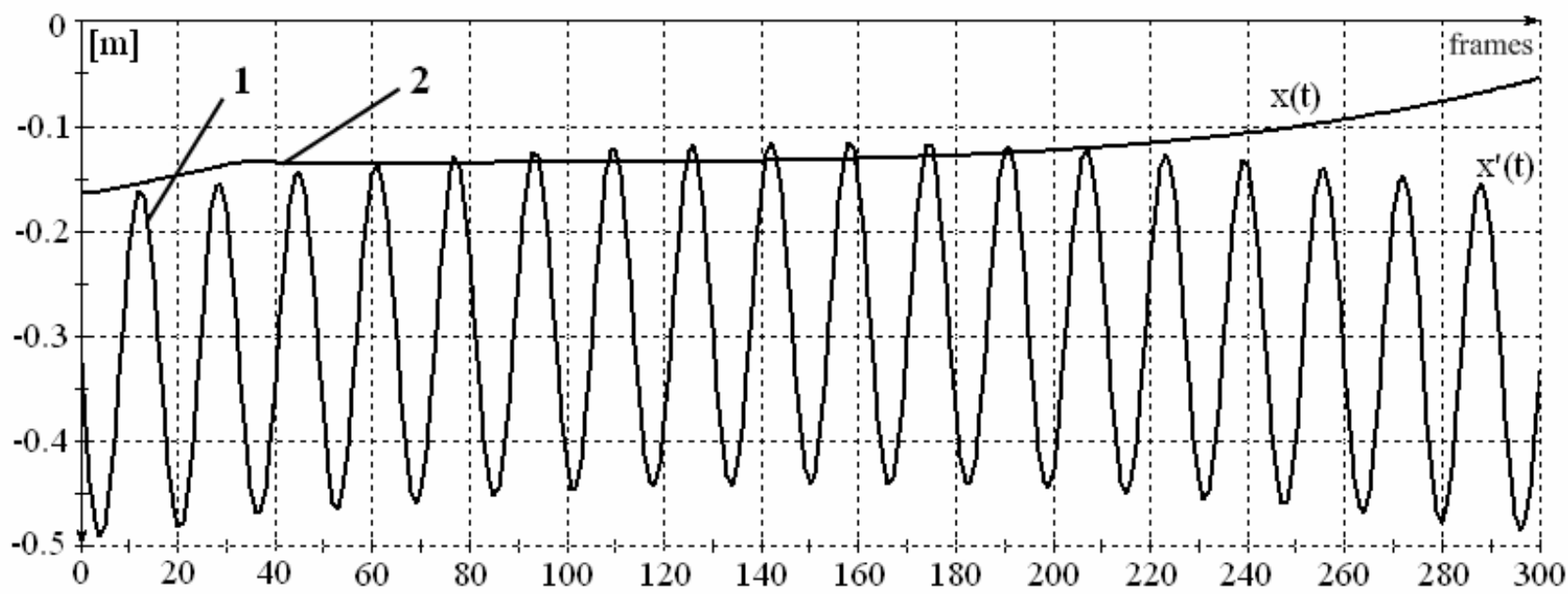

Fig. 8 - Controlled object oscillations: 1 - uncontrollable $x^{\prime}(t), 2$ - controllable $x(t)$

\section{CONCLUSION}

In this article the simulation model of the mechanical system stabilization by the active resonance control approach is considered. Simulation methodology and script of the AR control algorithm are developed. Virtual laboratory for simulations and investigations of control systems with controllers based on the AR approach is constructed. This virtual laboratory allows to investigate a control systems stabilization by the active resonance controller in case of low a priori information about the controlled process, to research the AR algorithm quality and stability dependences on initial conditions and parameters of mechanical models and environment. Constructed methodology allows to research different types of mechanical systems and external disturbances.

The active resonance algorithm for the first time is adapted and improved by using of numerical values of velocity and acceleration of a controlled object on each step of simulation for a system described by a second order differential equation.

Polynomial regression was implemented for an external disturbances equivalence extrapolation in the AR control algorithm for the mechanical system described by the differential equation (1). Efficiency of this regression for a system stabilization based on the active resonance approach were shown.

Obtained results will be applied for quantitative and qualitative estimations of the AR controller for control systems with random external disturbances.

\section{REFERENCES}

[1] M.I. Guchenko. Active resonance as a principle of operator reaction on the input signal in a compensatory tracking system, Interdepartmental scientific and technical journal
“System technology”, 2001, No. 4'(24), pp. 3038. [in Ukrainian]

[2] M.I. Guchenko. Active resonance stabilizing algorithm, Bulletin "New technologies" of economics and new technologies institute of J.I. Kravchenko, 2003, No.1(2), pp. 57-61. [in Ukrainian]

[3] V.M. Kuntsevich. Controlling in conditions of uncertainty: guaranteed results in tasks of control and identification. Naukova Dumka. Kiev, 2006. 264 p. [in Russian]

[4] Tutorial of WorkingModel Version 5.2 for Windows 95/98/ME/XP/NT 4.0, 2004.

[5] N.I. Guchenko, E.G. Slavko, M.J. Serik. Researches of disturbance signal modeling time influence on a controlled system stabilization accuracy, Bul. of Kremenchuk state polytechnic university, 2006, 2/2006 (37), pp. 12-15. [in Russian]

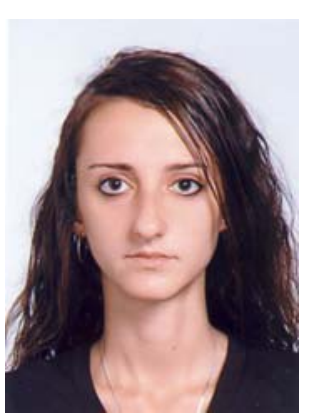

Olena Slavko, received the M.Sc. degree in Computer Engineering, Computer Systems and Networks from Kremenchuk Mykhaylo Ostrogradskiy state polytechnic university, Kremenchuk, Ukraine, in 2008. She is currently working toward the Ph.D. degree in Systems and Process Controlling, Kremenchuk Mykhaylo Ostrogradskiy State University. She is a part-time instructor, Computer and Information Systems Department, Kremenchuk Mykhaylo Ostrogradskiy State University. Her research focuses on automatic control in case of low a priori information about controlled processes. 